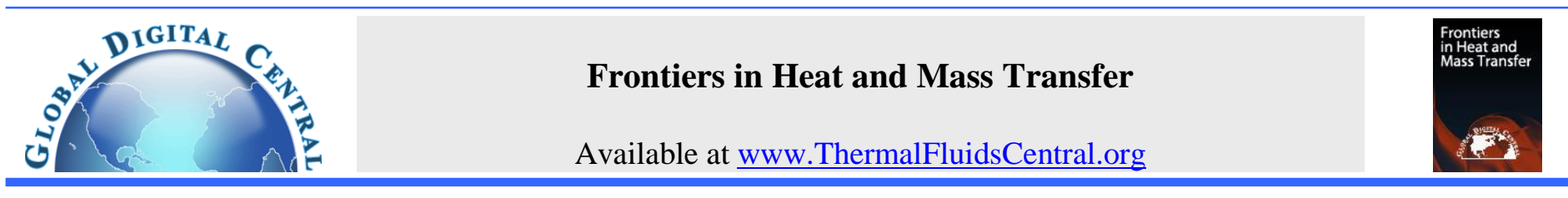

\title{
RELATIVE SLIP AMOUNT DEPENDENT ON THE INTERFACIAL SHEAR STRENGTH IN A NANO CHANNEL
}

\author{
Linyue Guan, Mingjun Pang, Hui Wang, Xuedong Jiang and Yongbin Zhang* \\ College of Mechanical Engineering, Changzhou University, Changzhou, 213164, Jiangsu Province, China
}

\section{ABSTRACT}

The paper presents an analysis for the relative slip amount related to the fluid-wall interfacial shear strength in a nano channel formed by two parallel smooth solid planes sliding against one another. A closed-form equation formulation for the relative slip amount was obtained. It was shown that the relative slip amount is constant in the inlet zone of the channel, where the interfacial slippage occurs. This substantiates the assumption made in the analysis of this kind of channel as shown in the study by Zhang (2015). It was also shown that with the increases of the channel height or the fluidwall interfacial shear strength, the interfacial slippage is reduced. The strong fluid-stationary wall interaction in the outlet zone of the channel results in more severe interfacial slippage than the medium-level fluid-stationary wall interaction in the outlet zone.
\end{abstract}

Keywords:Nano channel; Interfacial slippage; Interfacial shear strength; Interaction; Relative slip amount.

\section{INTRODUCTION}

In the study by Zhang (2015), an analysis was presented for a nano bearing formed between two parallel smooth solid planes respectively considering the fluid-wall interfaical slippage in the bearing inlet and outlet zones, by using the parameter of the relative slip amount $\left(\gamma_{\mathrm{s}}\right)$. In that analysis (Zhang, 2015), it was assumed that the relative slip amounts were respectively constant in the bearing inlet and outlet zones, and it was also phrased that the relative slip amount should be dependent on the fluid-wall interfacial shear strength.

In the past studies, the slip length (Gennes de, 2002; Spikes and Granick, 2003; Vinogradova, 1995) and the interfacial shear strength (Jacobson and Hamrock, 1984; Lee and Hamrock, 1990; Zhang, 2006a) were ever used to characterize the interfacial slippage. Based on these parameters, the interfacial slipping velocity can be derived. It was pointed out that the mechanism of the interfacial slippage is that the interfacial shear stress exceeds the interfacial shear strength, and the limiting shear strength model should be more convincible than the slip length model for describing the interfacial slippage ( Zhang, 2014).

The relative slip amount should be a third concept for describing the interfacial slippage. One of its advantage is the convenience of the analysis, particularly when it can be assumed as constant as done in the study by Zhang (2015). This parameter is obviously related to the interfacial slipping velocity (Zhang, 2015). The problem is that how to determine the magnitude of this parameter or why this parameter should be formulated as constant or other function dependent.

This paper gives an analysis for the formulation of the relative slip amount as dependent on the fluid-wall interfacial shear strength in the nano channel studied in the study by Zhang (2015), from the continuity of the mass flow rate through the channel. By this way, it can be seen that the relative slip amount is constant in the channel inlet zone, where the interfacial slippage occurs. This substantiates the assumption made in the study by Zhang (2015). As the relative slip amount directly measures the degree of the interfacial slippage, the influence of the operating condition on the interfacial slippage can be directly seen.

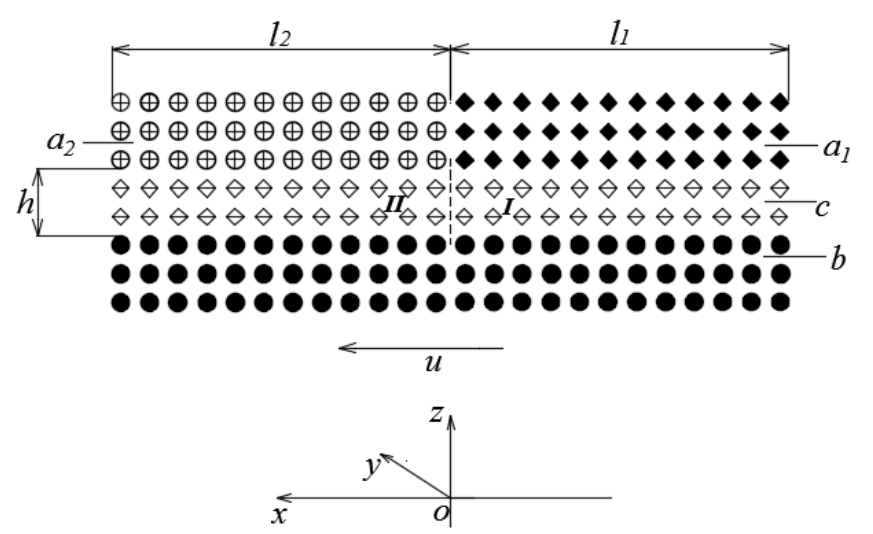

Fig. 1 The studied nano channel, coming from the study by Zhang (2015). a1, a2: stationary solid plane wall; $b$ : moving solid plane wall; $c$ : confined fluid; $I$ : inlet zone; $I I$ : outlet zone; $h$ :film thickness; $u$ : moving speed.

\section{STUDIED CHANNEL}

The studied channel was selected from the earlier research in the study by Zhang (2015) and is shown in Fig.1. It is formed between two parallel smooth plane walls. The upper wall is stationary and divided into the "a," and "a." sub-areas. Here, on the wall surfaces of these two sub-areas are respectively covered hydrophobic and hydrophilic coatings so that the confined film slips at the wall surface of the "a $a_{1}$ " 
sub-area and it does not slip at the wall surface of the "a2" sub-area. The lower wall is moving with the speed $u$ and uniform with a covered hydrophilic surface. The film does not slip on this surface. The channel is divided into the inlet and outlet zones, as shown in Fig.1.

\section{ANALYSIS}

The core of the present analysis is to derive the relative slip amount in the channel inlet zone defined in the study by Zhang (2015), which is dependent on the shear strength of the interface between the fluid and the upper wall surface in the channel inlet zone. The used coordinates are shown in Fig.1. The analysis is presented as follows.

\subsection{For the Inlet Zone}

The flow velocity of the confined film across the channel height in the inlet zone is (Zhang, 2013a):

$v=\frac{1}{2 \eta_{b f, I}^{e f f}} \frac{d p}{d x} z^{2}+c_{1} z+c_{2}$

where $\eta_{b f, I}^{e f f}$ is the effective viscosity of the confined film in the inlet zone, $p$ is the pressure, and $c_{1}$ and $c_{2}$ are constants.

From the boundary condition $\bar{u}_{b}=\left.v\right|_{z=0}=u$, it is obtained that $c_{2}=u$. Because of the interfacial slippage at the upper wall surface in the inlet zone, the magnitude of the shear stress at the upper wall surface in the inlet zone is equal to the fluid-wall interfacial shear strength $\tau_{s a}$ at that surface, and it is equated that (Zhang, 2013a):

$\theta_{\tau, a, I}\left(\frac{d p}{d x} h+c_{1} \eta_{b f, I}^{e f f}\right)=-\tau_{s a}$

where $h$ is the channel height and $\theta_{\tau, a, I}$ is the correction factor for the shear stress on the upper wall surface in the inlet zone (Zhang, 2006b). Thus,

$c_{1}=\left(-\frac{\tau_{s a}}{\theta_{\tau, a, I}}-\frac{d p}{d x} h\right) \frac{1}{\eta_{b f,{ }_{I}}^{e f f}}$

Substituting Eq. (3) and $c_{2}=u$ into Eq. (1) gives the film velocity at the upper wall surface in the inlet zone:

$\bar{u}_{a}=-\frac{\tau_{s a} h}{\theta_{\tau, a, I} \eta_{b f, I}^{e f f}}-\frac{1}{2 \eta_{b f, I}^{e f f}} \frac{d p}{d x} h^{2}+u$

Thus,

$\bar{l}_{I}=\frac{\bar{u}_{a}+\bar{u}_{b}}{2}=-\frac{1}{2 \eta_{b f, I}^{e f f}} \frac{\tau_{s a}}{\theta_{\tau, a, I}} h-\frac{1}{4 \eta_{b f, I}^{e f f}} \frac{d p}{d x} h^{2}+u$

The modified flow equation for the inlet zone is (Zhang, 2013a; Zhang, 2015):

$q_{m, b f}=\overline{l u}_{I} h \rho_{b f, I}^{e f f}+\frac{S_{I} \rho_{b f, I}^{e f f} h^{3}}{12 \eta_{b f, I}^{e f f}} \frac{d p(x)}{d x}$

where $q_{m, b f}$ is the mass flow rate per unit length through the channel, $\rho_{b f, I}^{e f f}$ is the average density of the confined film across the channel height in the inlet zone, and $S_{I}$ is the parameter depicting the noncontinuum effect of the confined film in the inlet zone.

Substituting Eq. (5) into Eq. (6) and rearranging gives:

$$
\frac{d p}{d x}=\frac{\eta_{b f, I}^{e f f}\left(q_{m, b f}-u h \rho_{b f, I}^{e f f}+\frac{\tau_{s a}}{\theta_{\tau, a, I}} \frac{1}{2 \eta_{b f, I}^{e f f}} h^{2} \rho_{b f, I}^{e f f}\right)}{\rho_{b f, I}^{e f f} h^{3}\left(\frac{S_{I}}{12}-\frac{1}{4}\right)}
$$

Integrating Eq. (7) gives:

$$
p=\frac{\eta_{b f, I}^{e f f}\left(q_{m, b f}-u h \rho_{b f, I}^{e f f}+\frac{\tau_{s a}}{\theta_{\tau, a, I}} \frac{1}{2 \eta_{b f, I}^{e f f}} h^{2} \rho_{b f, I}^{e f f}\right)}{\rho_{b f, I}^{e f f} h^{3}\left(\frac{S_{I}}{12}-\frac{1}{4}\right)} x+c_{3}
$$

Where $c_{3}$ is constant.

From the boundary condition $\left.p\right|_{x=-l_{1}}=0, c_{3}$ is solved from Eq. (8), and the pressure in the inlet zone is finally expressed as:

$$
p=\frac{\eta_{b f, I}^{e f f}\left(q_{m, b f}-u h \rho_{b f, I}^{e f f}+\frac{\tau_{s a}}{\theta_{\tau, a, I}} \frac{1}{2 \eta_{b f, I}^{e f f}} h^{2} \rho_{b f, I}^{e f f}\right)}{\rho_{b f, I}^{e f f} h^{3}\left(\frac{S_{I}}{12}-\frac{1}{4}\right)}\left(x+l_{1}\right) \quad \text { for }-l_{1} \leq x \leq 0
$$

The relative slip amount $\gamma_{s, I}$ in the inlet zone is defined by the following equation (Zhang, 2015):

$\bar{l} u_{I}=\frac{\left(\gamma_{s, I}+1\right) u}{2}$ gives:

Substituting Eq. (5) into Eq. (10) and eliminating $d p / d x$ by Eq. (7)

$$
\gamma_{s, I}=-\frac{\tau_{s a} h S_{I}}{\eta_{b f, I}^{e f f} \theta_{\tau, a, I} u\left(S_{I}-3\right)}-\frac{6 q_{m, b f}}{u h\left(S_{I}-3\right) \rho_{b f, I}^{e f f}}+\frac{S_{I}+3}{S_{I}-3}
$$

\subsection{For The Outlet Zone}

The modified flow equation for the outlet zone is (Zhang, 2013a; Zhang, 2015):

$q_{m, b f}=\frac{u}{2} h \rho_{b f, I I}^{e f f}+\frac{S_{I I} \rho_{b f, I I}^{e f f} h^{3}}{12 \eta_{b f, I I}^{e f f}} \frac{d p}{d x}$

where $\eta_{b f, I I}^{e f f}$ is the effective viscosity of the confined film in the outlet zone, $\rho_{b f, I I}^{e f f}$ is the average density of the confined film across the channel height in the outlet zone, and $S_{I I}$ is the parameter depicting the non-continuum effect of the confined film in the outlet zone.

Based on the boundary condition $\left.\quad p\right|_{x=l_{2}}=0$, it is solved from Eq. (12) that:

$p=\frac{\eta_{b f, I I}^{e f f}\left(12 q_{m, b f}-6 u h \rho_{b f, I I}^{e f f}\right)}{S_{I I} \rho_{b f, I I}^{e f f} h^{3}}\left(x-l_{2}\right) \quad$ for $\quad 0 \leq x \leq l_{2}$

According to the pressure continuity at $\mathrm{x}=0$, it is solved that (Zhang, 2015): 
$Q_{m, b f}=\frac{12 \psi \lambda_{s, I} C q_{I I} C y_{I I}-\frac{S_{I I} C q_{I I} \bar{\tau}_{s a}}{\theta_{\tau, a, I}}+2 S_{I I} C q_{I I} C y_{I}}{24 \lambda_{s, I} C y_{I I} \psi+2 \lambda_{q} S_{I I} C y_{I}}$

where $\quad Q_{m, b f}=q_{m, b f} /\left(u h \rho_{a}\right) \quad, \quad \psi=l_{2} / l_{1} \quad, \quad C q_{I}=\rho_{b f, I}^{e f f} / \rho_{a}$, $C q_{I I}=\rho_{b f, I I}^{\text {eff }} / \rho_{a} \quad, \quad C y_{I}=\eta_{b f, I}^{e f f} / \eta_{a} \quad, \quad C y_{I I}=\eta_{b f, I I}^{\text {eff }} / \eta_{a} \quad$, $\bar{\tau}_{s a}=\tau_{s a} h /\left(u \eta_{a}\right) \quad, \quad \lambda_{s, I}=S_{I} / 12-1 / 4 \quad, \quad$ and $\lambda_{q}=C q_{I I}\left(H_{I I}\right) / C q_{I}\left(H_{I}\right)$. Here, $\rho_{a}$ and $\eta_{a}$ are respectively the density and viscosity of the fluid at ambient condition when the fluid is continuum.

Substituting Eq. (14) into Eq. (11) yields:

$\gamma_{s, I}=-\frac{\bar{\tau}_{s a}}{C y_{I} \theta_{\tau, a, I}}\left(1+\frac{1}{4 \lambda_{s, I}}\right)-\frac{Q_{m, b f}}{2 \lambda_{s, I} C q_{I}}+\frac{1}{2 \lambda_{s, I}}+1$

It can be found from Eq. (15) that for a given channel, the relative silp amount $\gamma_{s, I}$ is constant, not varied with the coordinate $\mathrm{x}$. This substantiates the assumption made in the study by Zhang (2015) for the analysis derivation.

\subsection{Dimensionless Interfacial Slipping Velocity}

It is obtained from Eq. (10) that $\bar{u}_{a} / u=\gamma_{s, I}$. The interfacial slipping velocity at the upper wall surface in the inlet zone is (Zhang, 2013b):

$\Delta u_{a, x}=\bar{u}_{a}-u_{a}$

Since $u_{a}=0, \Delta u_{a, x}=\bar{u}_{a}$. The dimensionless interfacial slipping velocity at the upper wall surface in the inlet zone is thus:

$$
D U=\frac{\Delta u_{a, x}}{u}=\gamma_{s, I}
$$
2013b).

The interfacial slippage in the channel requires that DU $>0$ (Zhang,

\section{CALCUlation}

The parameters $C q_{I}\left(H_{I}\right)$ and $C q_{I I}\left(H_{I I}\right)$ are expressed as the following general form (Zhang, 2013a; Zhang, 2015):

$$
C q(H)=\left\{\begin{array}{l}
1 \quad \text { for } \quad H \geq 1 \\
m_{0}+m_{1} H+m_{2} H^{2}+m_{3} H^{3}, \text { for } 0<H<1
\end{array}\right.
$$

where $H=H_{I}$ or $H_{I I}$, and $m_{0}, m_{1}, m_{2}$ and $m_{3}$ are respectively constants. Here, $H_{I}=h / h_{c r, b f, I}$, and $H_{I I}=h / h_{c r, b f, I I}$.

The parameters $C y_{I}\left(H_{I}\right)$ and $C y_{I I}\left(H_{I I}\right)$ are expressed as the following general form (Zhang, 2013a; Zhang, 2015):

$C y(H)= \begin{cases}1 \quad, \quad \text { for } \quad H \geq 1 \\ a_{0}+\frac{a_{1}}{H}+\frac{a_{2}}{H^{2}} \quad, \quad \text { for } 0<H<1\end{cases}$

where $a_{0}, a_{1}$ and $a_{2}$ are respectively constants.

The parameters $S_{I}\left(H_{I}\right)$ and $S_{I I}\left(H_{I I}\right)$ are expressed as the following general form (Zhang, 2013a; Zhang, 2015):
$S(H)= \begin{cases}-1 \quad & \text { for } \quad H \geq 1 \\ {\left[n_{0}+n_{1}\left(H-n_{3}\right)^{n_{2}}\right]^{-1},} & \text { for } n_{3}<H<1\end{cases}$

where $n_{0}, n_{1}, n_{2}$ and $n_{3}$ are respectively constants.

The shear stress correction factor $\theta_{\tau, a, I}$ is formulated as (Zhang, 2013a):

$\theta_{\tau, a, I}\left(H_{I}\right)=k_{0}\left(H_{I}-1\right)^{2}+1 \quad$ for $0<H_{I} \leq 1$

where for the weak fluid-wall interaction in the inlet zone $k_{0}=-0.1$.

In the calculation, the interaction between the confined fluid and the wall in the "al" subzone is relatively weak, and the interaction between the confined fluid and the wall in the "a2" subzone is mediumlevel or relatively strong. In the calculation, $h_{c r, b f, I}=14 \mathrm{~nm}$, $h_{c r, b f, I I}=20 \mathrm{~nm}$ for the medium-level interaction between the confined fluid and the wall in the "a2" subzone, and $h_{c r, b f, I I}=40 \mathrm{~nm}$ for the relatively strong interaction between the confined fluid and the wall in the "a2" subzone (Zhang, 2015). The values of the other parameters in the calculation are respectively shown in Tables 1, 2 and 3 . The used symbols marking the interaction combinations are shown in Table 4.

Table 1 The values of the parameters in $C q_{I}\left(H_{I}\right)$ and $C q_{I I}\left(H_{I I}\right)$ (Zhang, 2013a; Zhang, 2015)

\begin{tabular}{|c|l|l|l|l|l|}
\hline \multicolumn{2}{|c|}{ Pq } & $m_{0}$ & $m_{1}$ & $m_{2}$ & $m_{3}$ \\
\hline \multirow{2}{*}{$C q_{I I}$} & $\begin{array}{l}\text { Strong } \\
\text { interaction }\end{array}$ & 1.43 & -1.723 & 2.641 & -1.347 \\
\cline { 2 - 6 } & $\begin{array}{l}\text { Medium } \\
\text { interaction }\end{array}$ & 1.30 & -1.065 & 1.336 & -0.571 \\
\hline \multicolumn{2}{|c|}{$C q_{I}$} & 1.116 & -0.328 & 0.253 & -0.041 \\
\hline
\end{tabular}

Table 2 The values of the parameters in $C y_{I}\left(H_{I}\right)$ and

\begin{tabular}{|c|c|c|c|c|}
\hline \multicolumn{2}{|c|}{$\begin{array}{ll}\mathrm{Cy} & \text { Parameter }\end{array}$} & $a_{0}$ & $a_{1}$ & $a_{2}$ \\
\hline \multirow{2}{*}{$C y_{I I}$} & $\begin{array}{l}\text { Strong } \\
\text { interaction }\end{array}$ & 1.8335 & -1.4252 & 0.5917 \\
\hline & $\begin{array}{l}\text { Medium } \\
\text { interaction }\end{array}$ & 1.0822 & -0.1758 & 0.0936 \\
\hline \multicolumn{2}{|l|}{$C y_{I}$} & 0.9507 & 0.0492 & $1.6447 \times 10^{-4}$ \\
\hline
\end{tabular}
$C y_{I I}\left(H_{I I}\right) \quad$ (Zhang, 2013a; Zhang, 2015)

Table 3 The values of the parameters in $S_{I}\left(H_{I}\right)$ and $S_{I I}\left(H_{I I}\right)$ (Zhang, 2013a; Zhang, 2015)

\begin{tabular}{|c|l|l|l|l|l|}
\hline \multicolumn{2}{|c|}{ S Parameter } & $n_{0}$ & $n_{1}$ & $n_{2}$ & $n_{3}$ \\
\hline \multirow{2}{*}{$S_{I I}$} & $\begin{array}{l}\text { Strong } \\
\text { interaction }\end{array}$ & 0.4 & -1.374 & -0.534 & 0.035 \\
\cline { 2 - 6 } & $\begin{array}{l}\text { Medium } \\
\text { interaction }\end{array}$ & -0.649 & -0.343 & -0.665 & 0.035 \\
\hline \multicolumn{2}{|l|}{$S_{I}$} & -0.1 & -0.892 & -0.084 & 0.1 \\
\hline
\end{tabular}

Table 4 The symbols used marking the interaction combinations (Zhang, 2016).

\begin{tabular}{|c|l|l|}
\hline $\begin{array}{c}\text { Fluid-stationary } \\
\text { wall interaction }\end{array}$ & II & I \\
\hline Symbols & Medium & Weak \\
\hline M-W & Strong & Weak \\
\hline S-W &
\end{tabular}




\section{RESULTS}

Figure 2(a) plots the values of $\gamma_{s, I}$ against the channel height for different interfacial shear strength $\bar{\tau}_{s a}$ when $\psi=5$ and the interaction type in the channel is S-W. It is shown that with the increases of the channel height or the interfacial shear strength $\bar{\tau}_{s a}$, the value of $\gamma_{s, I}$ is reduced, and this indicates the alleviation of the interfacial slippage occurring at the upper wall surface in the inlet zone.

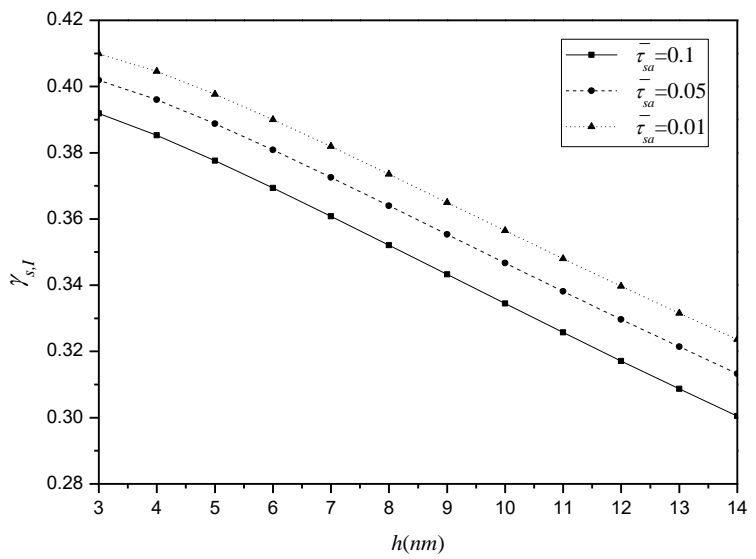

(a) For the $\mathrm{S}-\mathrm{W}$ interaction combination

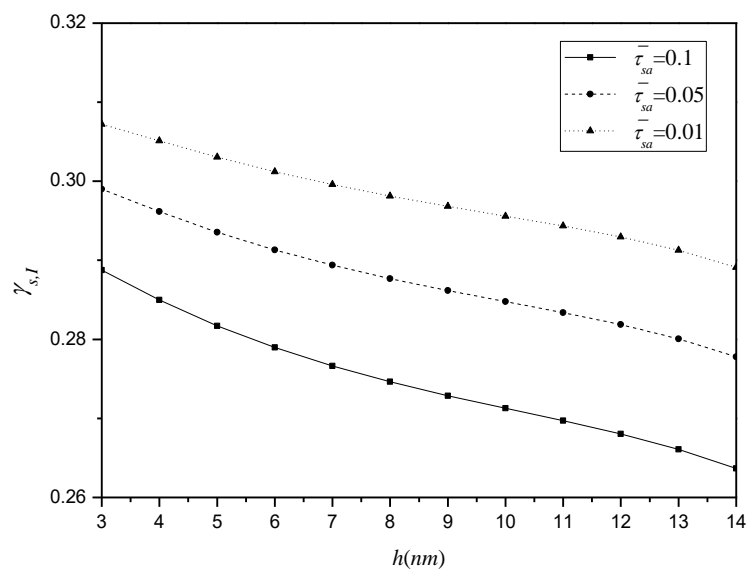

(b) For the M-W interaction combination

Fig. 2 Plots of the values of $\gamma_{s, I}$ against the channel height for different interfacial shear strength $\bar{\tau}_{s a}$ when $\psi=5$.

The obtained result agrees with the experimental observation on the wall slippage of the confined fluid in a nano gap which showed that with the reduction of the confining gap or with the weakening of the fluid-wall interaction, the wall slippage was increased (Churaev et al., 1984; Craig et al., 2001). Accroding to the values of $\gamma_{s, I}$, for the plotted cases, there occurs significant interfacial slippage at the upper wall surface in the channel inlet zone, and this should have a pronounced influence on the mass transfer in the channel. Figure 2(b) shows the results similar as in Fig.2(a) when $\psi=5$ and the interaction type in the channel is $\mathrm{M}-\mathrm{W}$.

Figure 3 plots the values of $\gamma_{s, I}$ against the dimensionless interfacial shear strength $\bar{\tau}_{s a}$ respectively for the S-W and M-W interaction types when $\psi=5$ and $\mathrm{h}=5 \mathrm{~nm}$. It is shown that with the increase of $\bar{\tau}_{s a}, \quad \gamma_{s, I}$ is linearly reduced for both of the interaction types, and for a given $\bar{\tau}_{s a}$ the S-W interaction combination generates a significantly greater interfacial slippage than the $\mathrm{M}-\mathrm{W}$ interaction combination. It indicates that a stronger fluid-wall interaction in the outlet zone will result in a more pronounced effect of the interfacial slippage.

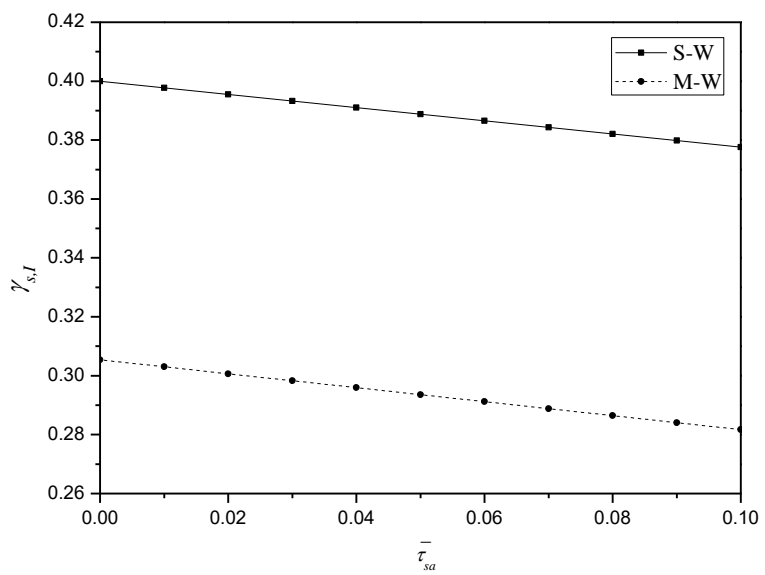

Fig. 3 Plots of the values of $\gamma_{s, I}$ against the dimensionless interfacial shear strength $\bar{\tau}_{s a}$ respectively for the S-W and M$\mathrm{W}$ interaction types when $\psi=5$ and $h=5 \mathrm{~nm}$.

\section{CONCLUSIONS}

The paper presents an analytical derivation of the relative slip amount $\gamma_{s, I}$ in the nano channel formed between two parallel smooth solid plane walls as studied in the study by Zhang (2015), when the interfacial slippage only occurs at the stationary wall surface in the channel inlet zone. The relative slip amount $\gamma_{s, I}$ is formulated as dependent on the fluid-wall interfacial shear strength $\bar{\tau}_{s a}$ at the stationary wall surface in the inlet zone.

It was shown that for a given channel, the value of $\gamma_{s, I}$ is constant in the whole area of the inlet zone. This substantiates the assumption made in the study by Zhang (2015) for the analysis derivation. It was also found from the obtained values of $\gamma_{s, I}$ that with the increases of $\bar{\tau}_{s a}$ or the channel height, the interfacial slippage is reduced; The $\mathrm{S}-\mathrm{W}$ interaction combination generates a greater interfacial slippage than the $\mathrm{M}-\mathrm{W}$ interaction combination in the studied channel.

\section{REFERENCES}

Churaev, N. V., Sobolev, V. D. and Somov, A. N.,1984, " Slippage of Liquids over Lyophobic Solid Surfaces," J. Colloid Interface Sci., 97, 574-581.

http://dx.doi.org/10.1016/0021-9797(84)90330-8

Craig, V. S. J., Neto, C. and Williams, D. R. M., 2001, "ShearDependent Boundary Slip in An Aqueous Newtonian Liquid," Phy. Rev. Lett., 87, 054504.

http://dx.doi.org/10.1103/PhysRevLett.87.054504

Gennes de, P. G., 2002, “On Fluid/Wall Slippage,” Langmuir, 18, 3413-3414.

http://dx.doi.org/10.1021/la0116342 
Jacobson, B. O. and Hamrock, B. J., 1984, "Non-Newtonian Fluid Model Incorporated into Elastohydrodynamic Lubrication of Rectangular Contacts," ASME J. Trib., 106, 275-284. http://dx.doi.org/10.1115/1.3260901

Lee, R. and Hamrock, B. J., 1990, “A Circular Non-Newtonian Fluid Model Part I: Used in EHL,"ASME J. Trib., 112,486-490. http://dx.doi.org/10.1115/1.2920285

Spikes, H. and Granick, S., 2003, "Equation for Slip of Simple Liquids at Smooth Solid Surfaces," Langmuir, 19, 5065-5071. http://dx.doi.org/10.1021/la034123j

Vinogradova, O. I., 1995, "Drainage of a Thin Liquid Film Confined between Hydrophobic Surfaces," Langmuir, 11, 2213-2220. http://dx.doi.org/10.1021/la00006a059

Zhang, Y. B., 2006a, "Contact-Fluid Interfacial Shear Strength and Its Critical Importance in Elastohydrodynamic Lubrication," Industr. Lubri. Trib., 58, 4-14.

http://dx.doi.org/10.1108/00368790610640064
Zhang, Y. B., 2006b, "Flow Factor of Non-Continuum Fluids in OneDimensional Contact," Industr. Lubri. Trib., 58, 151-169. http://dx.doi.org/10.1108/00368790610661999

Zhang, Y. B., 2013a, "The Reynolds Equation for Boundary Film Considering the Non-Continuum Effect and Its Application to The One-Dimensional Micro Step Bearing: Part II-Calculation for Boundary Slippage," J. Comput. Theor. Nanosci., 10, 609-615. http://dx.doi.org/10.1166/jctn.2013.2742

Zhang, Y. B., 2013b, "A Tilted Pad Thrust Slider Bearing Improved by Boundary Slippage,"Meccanica, 48, 769-781. http://dx.doi.org/10.1007/s11012-012-9630-6

Zhang, Y. B., 2014, "Review of Hydrodynamic Lubrication with Interfacial Slippage,” J. Balkan Trib. Assoc., 20, 522-538.

Zhang, Y. B., 2015, "Novel Nano Bearings Constructed by Physical Adsorption," Sci. Rep., 5, 14539.

http://dx.doi.org/10.1038/srep14539

Zhang, Y. B., 2016, 'Inhomogeneous Wall Surfaces for Designing Nano Slider Bearings,” J. Balkan Trib. Assoc., 22, 654-6. 